\title{
TABELAS DE VIDA DE ORIUS INSIDIOSUS (SAY, 1832) (HEMIPTERA: ANTHOCORIDAE) PREDANDO APHIS GOSSYPII GLOVER, 1877 (HEMIPTERA: APHIDIDAE) EM DIFERENTES CULTIVARES DE ALGODOEIRO
}

\section{S.A. De Bortolii ${ }^{1}$ J.E.M. Oliveira ${ }^{1}$, R.F. dos Santos ${ }^{1}$, L.C.P. Silveira ${ }^{2}$}

${ }^{1}$ UniversidadeEstadual Paulista, Faculdade deCiências Agráriase Veterinárias, Departamento de Fitossanidade, Laboratório de Biologia e Criação de Insetos, Via de Acesso Prof. Paulo Donato Castellane s/no, CEP14884-900, Jaboticabal, SP, Brasil. E-mail: eudes@fcav.unesp.br

\section{RESUMO}

\begin{abstract}
Os parâmetros de tabela de vida foram estudados para o predador Orius insidiosus em três cultivares de algodoeiro (Antares, CNPA7H e Acala 90 - respectivamente, glabra, de média pilosidade e pilosa) alimentando-se do pulgão-do-algodoeiro Aphis gossypii. O estudo mostrou que o tipo de cultivar pode influenciar nos parâmetros da tabela de vida do predador. A taxa intrínseca (rm) foi de 0,088 (Antares), 0,081 (CNPA7H) e 0,079 (Acala 90). Diferenças também foram observadas para o tempo de desenvolvimento, sobrevivência, fecundidade e longevidade entre os cultivares. A taxa líquida de reprodução (Ro) foi de 18,53 no cultivar Antares, mas com menor diferença nos cultivares CNPA7H $(12,26)$ e Acala $90(12,95)$. O tempo de desenvolvimento foi de 33,9; 30,62 e 32,13 dias para Antares, CNPA7H e Acala 90, respectivamente. A sobrevivência foi semelhante até o 12 dia em todos cultivares. Fêmeas de O. insidiosus sobreviveram até o 60 dia no cultivar Antares, sendo que começaram a ovipositar normalmente depois de dezesseis dias no cultivar Antares e dezenove dias em CNPA7H e Acala 90.
\end{abstract}

PALAVRAS-CHAVE: Controle biológico, Orius inidiosus, tabela de vida, Aphis gossypii, algodão.

\section{ABSTRACT}

LIFE TABLES AND FERTILITY OF ORIUS INSIDIOSUS (SAY) PREYING ON APHIS GOSSYPII GLOVER ON DIFFERENTS COTTON PLANT CULTIVARS. The life table parameters were studied for Orius insidiosus on the three cotton plant cultivars Antares, CNPA7H and Acala 90 (respectively, without tricome, medium tricome density and high tricome density). This study showed that the type of cultivar can strongly influence the life-table parameters. The intrinsic rate $(\mathrm{rm})$ was 0.088 (on Antares), 0.081 (CNPA7H) and 0.079 (on Acala 90), which was expected as these differences were also observed for development time, survival, fecundity and longevity on the cultivars. The reproductive rate (Ro) was 18.53 on the Antares cultivar, but showed less difference for CNPA7H (12.26) and Acala 90 (12.95). The development time was 33.9, 30.62 and 32.13 days for Antares, CNPA7H and Acala 90, respectively. The age-specific survival was similar until the 12th day. Maximum survival was 60 days on the Antares cultivar. Females usually began to oviposit after 16 days on the Antares cultivar and the after 19 days day on CNPA7H and Acala 90.

KEY WORDS: Biological control, minute pirate bug, Anthocoridae, life table.

\section{INTRODUÇÃO}

Entre as várias espécies que ocorrem na cultura do algodoeiro, são freqüentes as infestações do pulgão Aphis gossypii Glover, 1877 (Hemiptera: Aphididae), inseto que ocorre principalmente no início da cultura (CAUQuil, 1981; DenÉCHeRe, 1981). Essa espécie de pulgão é considerada a mais comum na cultura do algodoeiro, causando danos diretos através da sucção contínua de seiva (BRIOso, 1996)e danos indiretos pela transmissão de viroses (PEÑAMARTinez, 1992).

A cultura do algodoeiro apresenta-se como um excelente habitat para diversos inimigos naturais, dentreosquais se destacam os percevejos predadores do gênero Orius (CoLL, 1998). As espécies do gênero

\footnotetext{
${ }^{2}$ Universidade Federal de Lavras, Departamento de Entomologia, Lavras, MG, Brasil.
} 
Orius são pequenos predadores de ampla distribuição mundial, edeocorrência em diversas culturas, podendo exercer um papel fundamental na regulação da populaçãode pequenos artrópodes como tripes, ácaros, moscabranca, pulgões e ovos de lepidópteros (REZENDE, 1990; Lattin, 2000; Mendes; Bueno, 2001; LudwiG; Oetring, 2001; Argolo et al., 2002; STUdEBAKER; KRING, 2003).

A espécie Orius insidiosus é considerada generalista, possuindo habilidade de se alimentar de diferentes presas e substratos e de se abrigar em diferentes agroecossistemas, o que a torna apta à exploração do ecossistema e a sobreviver naturalmente (Silveira et al., 2003). Esses percevejos possuem certas características queos tornam promissores agentes de controle biológico, destacando-se a alta eficiência de busca, habilidade para aumentar a população eagregar-se rapidamente quando há presas em abundância, além de sobreviver em baixa densidade de presas (Bush et al., 1993).

O tipo de alimento pode interferir em vários parâmetros desse predador, como sobrevivência, longevidade, fecundidade e viabilidade dos ovos, podendo inclusive levá-lo a não completar o desenvolvimento (RICHARDS; SCHMIDT, 1996; BUENO, 2000). A fecundidade de $O$. insidiosus é diretamente afetada pelo alimento, sendo esse um fator de influência direta para o aumento rápido de sua população(KIMAN; YEARGAN, 1985; RiCHARDS; SCHMIDT, 1996).

As fêmeas de $O$. insidiosus ovipositam endofiticamenteemuma grandevariedadedesubstratos naturais, como vagens deleguminosas, caules defeijão, brotos de batata, inflorescências de picão (Bidens pilosa L.), pecíolos de folhas de algodoeiro, folhas de gerânio, pepino, batateira, entre outros (BUENO, 2000). Assim, o sucesso do desenvolvimento desses percevejos pode ser influenciado por diversosfatoresquepoderãointerferir na sua capacidade predatória.

Estudos a respeito de respostas sobre a história de vida sãonecessários para conhecimento doartropóde em foco (SoutHWood, 1978). Dessa forma, objetivando quantificar os parâmetros de tabela de vida de fertilidade e de esperança de vida, justifica-se a realização de estudos sobre bioecologia de $O$. insidiosus em agroecossistema do algodoeiro, tendo como presa o pulgão A. gossypii.

\section{MATERIAL E MÉTODOS}

O experimento foi conduzido no Laboratório de Biologia e Criação de Insetos do Departamento de Fitossanidade da Faculdade de Ciências Agrárias e Veterinárias da Universidade Estadual Paulista FCAV/UNESP, Jaboticabal, São Paulo. As condições experimentais foram de $25 \pm 1^{\circ} \mathrm{C}, 70 \pm 10 \%$ de umidade relativa e $14 \mathrm{~h}$ de fotofase.
Plantio de algodoeiro. Plantas de algodoeiro (Gossypium hirsutum L. raça Latifolium Hutch), cultivares Acala 90, CNPA 7H e Antares foram cultivadas em bandejasdeisoporemantidasemteladoslivresdepragas e inimigos naturais. Foram realizados plantios escalonados com intervalos de 10 dias para obtenção de folhas e plantas adequadas para a manutenção da criação do pulgão $A$. gossypii e condução do experimento.

Criação de Orius insidiosus. A criação do predador $O$. insidiosus foi iniciada a partir de espécimes coletados em agroecossistemas de algodão e milho no Campus da FCAV/UNESP, Jaboticabal, SP. A criação foi mantida em câmara climatizada tipo B.O.D. ajustada a $25 \pm 1^{\circ} \mathrm{C}$ e $70 \pm 10 \%$ de umidade relativa e $14 \mathrm{~h}$ defotoperíodo, adotando-se a metodologia adaptada de IsENHOuR; YeArGAN (1981), Schmidt et al. (1995), Bueno (2000) e Silveira; Bueno (2003).

Os adultos de O. insidiosus foram mantidos em gaiolas (1,7 L) vedadas com filme de PVC. No interior da gaiola colocou-se papel toalha, servindo como abrigo para os predadores. Para promover aeração foram feitos furos no filme de PVC com auxílio de um estilete. Em cada recipiente de criação foram mantidos 50 casais do percevejo predador, ovos de Anagasta kuehniella (Zeller, 1879) (Lepidoptera: Pyralidae) como fonte alimentar e inflorescências de Bidens pilosa L. (Asteraceae) como substrato de oviposição, as quais foram tratadas em solução de hipoclorito a $2 \%$ por cerca de 2 minutose, após secagem foram fixadas a um chumaço dealgodão embebidoemágua destilada para a manutenção da turgescência e fornecimento da umidade aos predadores. As inflorescências contendo ovos do predador foram removidas e transferidas para placas de Petri $(14 \times 2 \mathrm{~cm})$ fechadas com filme de PVC.

Um dia antes do previsto para eclosão das ninfas, ovos de A. kuehniella foram colocados no topo da inflorescência como fonte de alimento para as ninfas recém-eclodidas. As ninfas do predador ao eclodirem permaneceram no interior da mesma placa por todo período ninfal. No interior da placa foi colocado papel toalha, servindo como abrigo para os predadores. Para promover aeração foram feitos furos no filme dePVC, com auxílio de um estilete. Emcada recipiente de criação das ninfas foram mantidos cerca de 100 indivíduos, sendo o fornecimento de água feito por um chumaço de algodão embebido com água destilada. O alimento e água foram renovados a cada dois dias. Os adultos, logo após a emergência, foram separados em casais para iniciar o novo ciclo da criação.

Criação de Aphis gosypii. Os pulgões utilizados nos experimentos foram oriundos da criação mantida no laboratório em plantas do cultivar IAC 24. Essa criação foi iniciada com indivíduos coletados em plantas de algodoeiro em plantios comerciais da região de Jaboticabal, SP, sendo, posteriormente, transferidos para plantas mantidas em telado e condições de labo- 
ratório, protegidas para evitar a migração e infestação de outras espécies de pulgões e de inimigos naturais. Periodicamente, as colônias dos pulgões foram transferidas para novas plantas, com 20 dias de emergência (Oliveira et al., 2005; De Bortoli; Oliveira, 2005).

O efeito das diferentes cultivares de algodoeiro foi verificado quanto à presença e número de tricomas. Os testes foram conduzidos utilizando-se as cultivares Antares, CNPA7H e Acala 90, respectivamente, glabra, de pilosidade média e pilosa, sobre os aspectos biológicos e a capacidade predatória de $O$. insidiosus sobre o pulgão do algodoeiro A. gossypii.

Fase jovem. $O$ experimento foi iniciado com 75 ninfas de primeiroínstar, oriundas de criação mantida em laboratório. As ninfas foram agrupadas em cinco por gaiola (placas de Petri-14 $\times 2 \mathrm{~cm}$ ) contendo no seu interior uma folha de algodoeiro de cada cultivar, inserida num tubo com água (tipo anestésico odontológico) com um chumaço de algodão. Diariamente foram disponibilizados em cada tratamento/ cultivar, quinze pulgões de terceiro/quarto estádio e, a cada $24 \mathrm{~h}$ da oferta, os insetos, predados, ou não foram repostos ou substituídos por indivíduos de idade semelhante ao descrito. As avaliações foram realizadas a cada 24 h, observando-se a duração e sobrevivência em cada ínstar e total da fase ninfal.

Fase adulta. Ao atingirem a fase adulta, as fêmeas de $O$. insidiosus provenientes das ninfas mantidas nos seus respectivos tratamentos foram acasaladas, mantendo-se os machos durante três dias (período suficiente para efetivação da cópula). As fêmeas de $O$. insidiosus foram individualizadas em placas de Petri - $14 \times 2 \mathrm{~cm}$ contendo no seu interior uma folha de algodoeiro de cada cultivar, inserida num tubo com água (tipo anestésico odontológico) com um chumaço de algodão. Diariamente foram disponibilizados em cada tratamento/cultivar, quinze pulgões de terceiro/quarto estádio e, a cada 24 h da oferta, os insetos, predados ou não, foram repostos ou substituídos por indivíduos deidade semelhante ao descrito. Avaliousea longevidade e reprodução, o número total de ovos e de ninfas por fêmea.

Foram elaboradas tabelas de vida de fertilidade e de esperança de vida para fêmeas de O. insidiosus, de acordo com SouTHWOOD (1978), sendo os parâmetros determinados como: taxa líquida de reprodução (Ro $=\sum($ lx.mx $\left.)\right)$, tempo de geração $\left[\mathrm{T}=\sum\right.$ (lx.mx.x) $/ \sum$ (lx.mx)], taxa intrínseca de crescimento natural ( $\mathrm{rm}=$ $\ln (\mathrm{Ro}) / \mathrm{T})$ e taxa finita de aumento populacional $(\lambda$ $=$ anti $\log (\mathrm{rm} .0,4343))$, sendo $x$, idade específica em dias, lx a sobrevivência diária a partir de ovos e $\mathrm{mx}$, a produção diária de fêmeas.

\section{RESULTADOS E DISCUSSÃO}

A partir do número de descendentes fêmeas obtido, foram construídas as tabelas de vida de fertilidade e de esperança de vida paraO. insidiosus, mantidonos três cultivares de algodeiro, predando o pulgão $A$. gossypii (Tabelas 1, 2 e 3).

A oviposição começou no 16 dia (três dias após transformação em adultos) para fêmeas deO.insidiosus mantidas nocultivar Antares eno19-paraas mantidas nos cultivares CNPA7H e Acala 90, contado a partir da eclosão das ninfas (Fig. 2). TommAsini et al. (2004) observaram período similar (16 dias) para o início da oviposição das fêmeas de $O$. insidiosus quando alimentadas com Frankliniella occidentalis (Pergande, 1895) (Thysanoptera: Thripidae). Em estudos semelhantes, Bush et al. (1993) e Mendes et al. (2003) observaram que as fêmeas de $O$. insidiosus predando o pulgão $A$. gossypii iniciaram sua oviposição com dezesseis dias a partir da eclosão das ninfas.

A partir das ninfas iniciamente mantidas nos respectivos tratamentos, determinou-se a viabilidade dos indivíduos que atingiram a fase adulta, que foi de 60 , 56 e $57 \%$ nos cultivares Antares, CNPA7H e Acala 90, respectivamente (Tabela 1 ). As fêmeas de O.insidiosus produziram em média 33,07 ovos no cultivar Antares; 23,30 ovos no CNPA7H e 22,27 ovos no Acala 90, com viabilidade de 71,$5 ; 70,0$ e $69,8 \%$, respectivamente. Tommasin et al. (2004) relataram que fêmeas de $O$. insidiosus, quando alimentadas comF.occidentalis,produziramodobrodeovoscomparando-sea esseestudo. Mendes etal. (2003) relatam que fêmeas deO.insidiosus ovipositaram de 2,0 a 53,4 ovos/dia, quando a densidade de $A$. gossypii variou de 10 a 60 ninfas.

Tabela 1 - Somatória dos dados da tabela de vida de fertilidade para fêmeas deOrius insidiosus criadas com Aphis gossypii em diferentes cultivares de algodoeiro. Temp. $25 \pm 1^{\circ}$ C; UR $70 \pm 10 \%$; fotofase 12h, Jaboticabal, 2006.

\begin{tabular}{|c|c|c|c|c|c|c|c|c|c|c|c|c|}
\hline \multirow[b]{2}{*}{$X$} & \multicolumn{4}{|c|}{ Antares } & \multicolumn{4}{|c|}{ CNPA7H } & \multicolumn{4}{|c|}{ Acala 90} \\
\hline & $1 x$ & $\mathrm{mx}$ & $\operatorname{mxlx}$ & $\operatorname{mxlxx}$ & $1 x$ & $\mathrm{mx}$ & $\operatorname{mxlx}$ & $\operatorname{mxlxx}$ & $1 x$ & $\mathrm{mx}$ & $\operatorname{mxlx}$ & $\operatorname{mxl} x x$ \\
\hline$\Sigma$ & 24,91 & & 18,53 & 616,89 & 22,79 & & 12,26 & 375,42 & 23,05 & & 12,95 & 416,19 \\
\hline
\end{tabular}




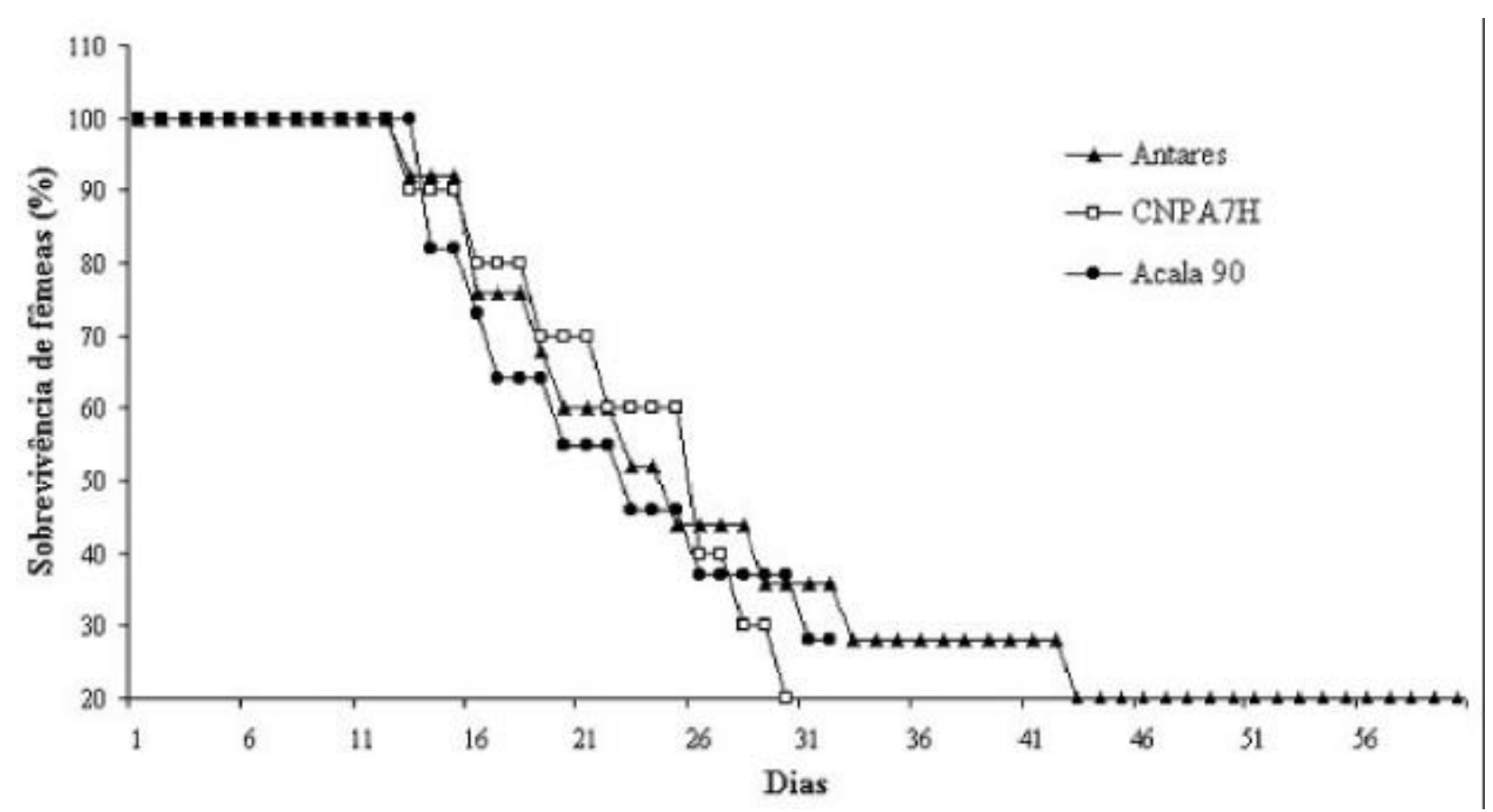

Fig. 1 - Sobrevivência de fêmeas de Orius insidiosus em três cultivares de algodoeiro e alimentados com ninfas de Aphis gossypii. Temp. $25 \pm 1^{\circ}$ C; UR $70 \pm 10 \%$; fotofase 12h, Jaboticabal, 2006.

Tabela 2 - Parâmetros da tabela de vida de fertilidade de fêmeas de Orius insidiosus criadas com Aphis gossypii em diferentes cultivares de algodoeiro. Temp. $25 \pm 1^{\circ} \mathrm{C}$; UR $70 \pm 10 \%$; fotofase 12h, Jaboticabal, 2006 .

\begin{tabular}{llcl}
\hline Características & \multicolumn{3}{c}{ Tratamentos } \\
\cline { 2 - 4 } & Antares & CNPA7H & Acala 90 \\
\hline Ro & 18,53 & 12,26 & 12,95 \\
$\mathrm{~T}$ & 33,29 & 30,62 & 32,13 \\
$\mathrm{Rm}$ & 0,088 & 0,081 & 0,079 \\
$\lambda$ & 1,09 & 1,08 & 1,08 \\
\hline
\end{tabular}

Ro - Taxa Líquida de Reprodução $\left(\sum=\mathrm{mx} .1 \mathrm{x}\right)$;

$\mathrm{T}$ - Tempo Médio de Geração ( $\mathrm{T}=\sum$ lx.mx.x / $\sum$ lx.mx); rm - Taxa infinitesimal (intrínseca) de aumento populacional ( $\mathrm{rm}=\log$ Ro/T. 0,4343);

$\lambda$-Taxa Finita de Aumento populacional $[\lambda=\operatorname{anti} \log (\mathrm{rm}$. $0,4343)]$.

A taxa líquida de reprodução (Ro) (números de descendentes fêmeas que darão origem a fêmeas reprodutivas, no decorrer de uma geração) foi maior no cultivar Antares (Ro $=18,53)$, indicando que os demais cultivares (CNPA7H e Acala 90) exercem forte influência sobre a taxa de desenvolvimento e mortalidade de O. insidiosus. Entretanto, os valores da taxa de reprodução (Tabela 2 ) revelam um crescimento populacional positivo para $O$. insidiosus em todos os cultivares. Menor valor para a taxa líquida de reprodução $(\operatorname{Ro}=17,9)$ foi observado para $O$. insidiosus tendo como presaF.occidentalis (TOMMASINI et al., 2004).

Otempo médio de geração (T) (tempo decorrido do nascimento dos pais até o de seus descendentes) foi de 33,29; 30,62 e 32,13 dias, nos cultivares Antares, CNPA7He Acala 90, respectivamente. TOMmAsini etal. (2004) obtiveram tempo médio de geração (T) semelhante (33,6 dias) paraO. insidiosus tendo como presa F. occidentalis.

A razão infinitesimal de aumento populacional (rm) foi de 0,088 nocultivar Antares, 0,081 no CNPA7H ede 0,079 no cultivar Acala 90 (Tabela 2), verificandose que essa diferença chega a ser de $10,3 \%$ entre o cultivar Antares e Acala 90.

A razão finita de aumento populacional $(\lambda)$ (número de fêmeas adicionadas à população, por fêmeas, por unidade de tempo) foi de 1,09 no cultivar Antares e de 1,08 nos CNPA7He Acala 90 (Tabela 2), sendo que esses valores revelam a agregação de mais de um indivíduo por fêmea, de uma geração para outra.

A curva de sobrevivência para O. insidiosus apresentou uma queda inicial no início da idade (12 dias) em todos cultivares (Fig. 1), ocorrendo em seguida certa freqüência na proporção de mortalidade com o aumento da idade dos insetos, seguindo o mesmo comportamento até o final da fase.

Na fase adulta os insetos passaram por um período de dois dias a partir da data do acasalamento sem reproduzir(pré-oviposição), seguidodafasereprodutiva, onde inicialmente o esforço reprodutivo foi alto a partir 
do quarto dia de oviposiçãoe declinando com oenvelhecimento dos insetos (Fig. 2) onde a reprodução iniciouseno 20dia, começandoa decrescer efinalizandono60응 dia. Nos cultivares CNPA e Acala 90, aparentemente, houve uma resistência inicial das fêmeas para oviposição, notando-sequeoesforçoreprodutivosedeu no 26ㅇdia(Fig. 2). Operíodo deoviposição foi de 44 dias, quando O. insidiosus foialimentadocomovos de Anagasta kuehniella (MENDEs et al., 2002).
A tabela de esperança de vida revelou que fêmeas de O. insidiosus podem viveraté 60 diasquandomantidas no cultivar Antares (Tabela 3). Para os primeiros dez dias obteve-se uma esperança de vida de 47,3 dias, com risco de $7,7 \%$ de tal fato não ocorrer. Nos cultivares CNPA7He Acala 90 a esperança de vida do predador foi, respectivamente, de 38,0 e 32,3 dias. Para todos cultivares esperança de vida de $O$. insidiosus tendeu a diminuir à medida que o predador envelheceu.

Tabela 3 - Tabela de esperança de vida para fêmeas deOriusinsidiosus criadas com Aphis gossypii em diferentes cultivares de algodoeiro. Temp. $25 \pm 1^{\circ}$ C; UR $70 \pm 10 \%$; fotofase $12 \mathrm{~h}$, Jaboticabal, 2006.

\begin{tabular}{|c|c|c|c|c|c|c|c|c|}
\hline \multirow[t]{2}{*}{ Classes de idade } & $x$ & $\mathrm{Lx}$ & $\mathrm{dx}$ & Ex & $\mathrm{qx}$ & Sx & $\mathrm{Tx}$ & Ex \\
\hline & \multicolumn{8}{|c|}{ Antares } \\
\hline $0-5$ & 0 & 13 & 0 & 13,0 & 0,000 & 1,00 & 61,5 & 4,73 \\
\hline $6-10$ & 6 & 13 & 1 & 12,0 & 0,077 & 0,923 & 48,5 & 3,73 \\
\hline $11-15$ & 11 & 11 & 2 & 10,0 & 0,182 & 0,818 & 36,5 & 3,32 \\
\hline $16-21$ & 16 & 9 & 2 & 7,5 & 0,222 & 0,778 & 26,0 & 2,89 \\
\hline $21-25$ & 21 & 6 & 3 & 5,0 & 0,50 & 0,50 & 18,5 & 3,08 \\
\hline $26-30$ & 26 & 4 & 2 & 3,5 & 0,50 & 0,50 & 13,5 & 3,37 \\
\hline $31-35$ & 31 & 3 & 1 & 3,0 & 0,33 & 0,67 & 10,0 & 3,33 \\
\hline $36-40$ & 36 & 3 & 0 & 2,5 & 0,00 & 1,00 & 7,0 & 2,33 \\
\hline $41-45$ & 41 & 2 & 1 & 1,5 & 0,50 & 0,50 & 4,5 & 2,25 \\
\hline $46-50$ & 46 & 1 & 0 & 1,0 & 0,00 & 1,00 & 3,0 & 3,00 \\
\hline $51-56$ & 51 & 1 & 0 & 1,0 & 0,00 & 1,00 & 2,0 & 2,00 \\
\hline \multirow[t]{2}{*}{$56-60$} & 56 & 1 & 1 & 1,0 & 1,00 & 0,00 & 1,0 & 1,00 \\
\hline & \multicolumn{8}{|c|}{ CNPA7H } \\
\hline $0-5$ & 0 & 10 & 0 & 10,0 & 0,000 & 1,00 & 38,0 & 3,80 \\
\hline $6-10$ & 6 & 10 & 0 & 9,0 & 0,000 & 1,00 & 28,0 & 2,80 \\
\hline $11-15$ & 11 & 8 & 2 & 6,5 & 0,25 & 0,75 & 19,0 & 2,37 \\
\hline $16-21$ & 16 & 5 & 3 & 6,0 & 0,60 & 0,40 & 12,5 & 2,5 \\
\hline $21-25$ & 21 & 1 & 1 & 2,5 & 0,50 & 0,50 & 6,5 & 6,5 \\
\hline \multirow[t]{2}{*}{$26-30$} & 26 & 4 & 4 & 2,0 & 1,00 & 0,00 & 4,0 & 1,0 \\
\hline & \multicolumn{8}{|c|}{ Acala 90} \\
\hline $0-5$ & 0 & 11 & 0 & 11,0 & 0,000 & 1,00 & 35,5 & 3,23 \\
\hline $6-10$ & 6 & 11 & 0 & 9,5 & 0,000 & 1,00 & 24,5 & 2,58 \\
\hline $11-15$ & 11 & 8 & 3 & 6,5 & 0,375 & 6,25 & 15,0 & 2,31 \\
\hline $16-21$ & 16 & 5 & 3 & 3,5 & 0,60 & 0,40 & 8,5 & 2,43 \\
\hline $21-25$ & 21 & 2 & 2 & 3,0 & 1,00 & 0,00 & 5,0 & 1,66 \\
\hline $26-30$ & 26 & 1 & 1 & 1,0 & 1,00 & 0,00 & 2,0 & 0,50 \\
\hline $31-35$ & 31 & 1 & 1 & 1,0 & 1,00 & 0,00 & 1,0 & 1,0 \\
\hline
\end{tabular}

$\mathrm{X}$ - Intervalo de idade;

Lx - Número médio de sobreviventes no início da idade $X$;

dx - Número de indivíduos mortos durante o intervalo etário X (mortes no período);

Ex - Estrutura etária (número de indivíduos vivos entre uma classe e outra);

qx - Razão de mortalidade por intervalo de idade ( $q x=d x / L x)$;

Sx - Razão de sobrevivência ( $\mathrm{Sx}=1-\mathrm{qx})$;

Tx - Número total de insetos de idade $x$ além da idade $x$;

ex - Expectativa de vida para os indivíduos de idade $\mathrm{x}(\mathrm{Tx} / \mathrm{Lx})$; 

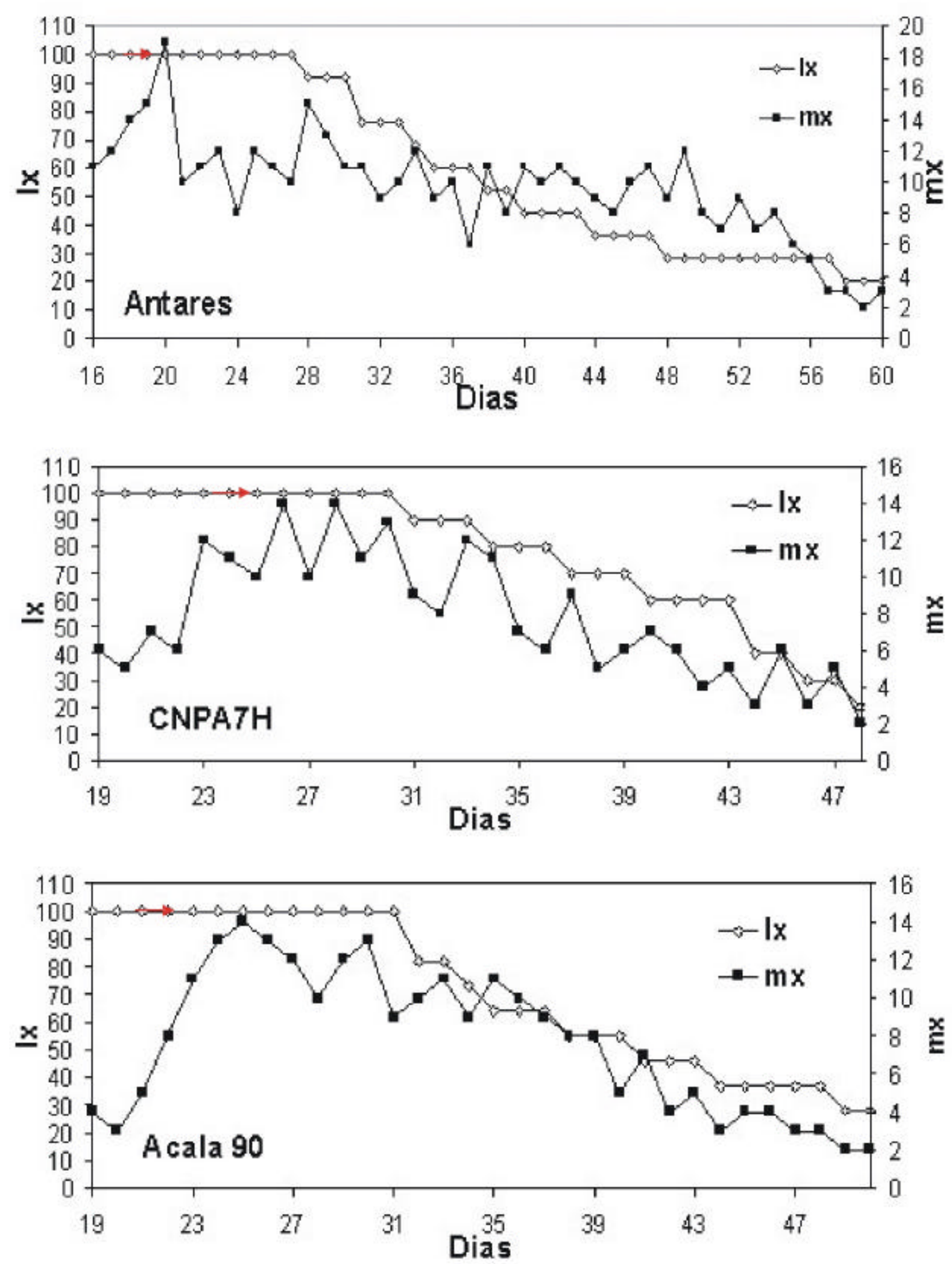

Fig. 2 - Relação entre fertilidade especifica $(\mathrm{mx})$ e taxa de sobrevivência (lx) de Orius insidiosus predando Aphis gossypii em três cultivares de algodoeiro. A seta indica a taxa máxima de aumento. Temp. $25 \pm 1^{\circ} \mathrm{C}$; UR $70 \pm 10 \%$; fotofase $12 \mathrm{~h}$, Jaboticabal, 2006. 
Tabelas de vida de Orius insidiosus (Say, 1832) (Hemiptera: Anthocoridae) predando Aphis gossypii Glover, 1877 (Hemiptera: Aphididae) em diferentes cultivares de algodoeiro.

\section{CONCLUSÕES}

1. Fêmeas de O. insidiosus ovipositam mais cedo e maior número de ovos quando mantidas no cultivar Antares.

2. A taxa de reprodução, o tempo médio de geração, a razão infinitesimal de aumento populacional e a expectativa de vida são maiores no cultivar Antares.

3. A razão finita de aumento populacional é superior a um, o que revela a agregação de mais de um indivíduo por fêmea, de uma geração para outra.

\section{AGRADECIMENTOS}

À FAPESP - Fundação de Amparo à Pesquisa do Estado de São Paulo, pelo suporte financeiro e bolsa concedida a José Eudes de Morais Oliveira (Proc. n. 03/11924-7); ao Prof. Dr. José Carlos Barbosa (FCAV/ UNESP), pelo auxílio na elaboração do delineamento experimental e análise dos dados.

\section{REFERÊNCIAS}

ARGOLO, V.M.; BUENO, V.H.P.; SILVEIRA, L.C.P. Influência do fotoperíodo na reprodução e longevidade de Orius insidiosus (Say) (Heteroptera: Anthocoridae). Neotropical Entomology, v.31, n.2, p.257261, 2002.

BRIOSO, P.S.T. Doenças causadas por vírus em pimentão. Informe Agropecuário, v.18, n.184, p.74-80, 1996. BuENo, V.H.P. Desenvolvimento e multiplicação de percevejos predadores do gênero Orius Wolff. In: (Ed.). Controle biológico de pragas: produção massal e controle de qualidade. Lavras: UFLA, 2000. p.69-90.

BUSH, L.; KRING, T.J.; RUBSERSON, J.R. Suitability of greenbugs, cotton aphids, and Heliothis virescens eggs for the development and reproduction of Orius insidiosus. Entomologia Experimentalis et Applicata, v.67, n.2, p.217-222, 1993 .

CAUQUIL, J. Récents développements dans la lutte contre la maladie bleue du cotonnier en Afrique Centrale. Coton et Fibres Tropicales, v.36, n.2, p.297-304, 1981.

COLL, M. Living and feeding on plants in predatory heteroptera. In: COLL, M.; RUBERSON J.R. (Ed.). Predatory Heteroptera: their ecology and use in biological control. Lanham: Thomas Say, 1998. p.89129.

DE BORTOLI, S.A.; OLIVEIRA, J.E.M. Estudos com pulgão vetor de vírus. Informativo do Manejo Ecológico de Pragas, n.42, p.492, 2005.
DENÉCHÈRE, M. Note sur la distribution et l'évaluation des populations d'Aphis gossypii Glov. (Hymenoptère, Aphididae) sur cotonniers en République Centrafricaine. Cotton et Fibres Tropicales, v.36, n.2, p.271-280, 1981.

ISENHOUR, D.J.; YEARGAN, K.V. Effect of crop phenology on Orius insidiosus populations on stripcropped soybean and corn. Journal of the Georgia Entomological Society, v.16, n.3, p.310-322, 1981.

KIMAN, Z.B.; YEARGAN, K.V. Development and reproduction of the predator Orius insidiosus (Hemiptera: Anthocoridae) reared on diets of selected plant material and arthropod prey. Annals of the Entomological Society of America, v.78, n.4, p.464-467, 1985.

LATTIN, J.D. Economic importance of minute pirate bugs (Anthocoridae). In: SCHOEFER, C.W.S.; PANIZZI, A.R. (Ed.). Heteroptera of economic importance. Florida: CRC Press, 2000. 828p.

LUDWIG, S.; OETTING, R. Effect of spinosad on Orius insidiosus (Hemiptera: Anthocoridae) when used for Frankliniella occidentalis (Thysanoptera: Thripidae) control on greenhouse pot chrysanthemums. Florida Entomologist, v.84, n.2, p.311-313, 2001.

MENDES, S.M.; BUENO, V.H.P; ARGOLO, V.M.; SILVEIRA, L.C.P. Type of prey influences biology and consumption rate of Orius insidiosus (Say) (Hemiptera: Anthocoridae). Revista Brasileira de Entomologia, v.46, n.1, p.99-103, 2002.

MENDES, S.M.; BUENO, V.H.P.; CARVALHO, L.M.; SILVEIRA, L.C.P. Efeito da densidade de ninfas de Aphis gossypii Glover, 1877 (Hemiptera, Aphididae) no consumo alimentar e aspectos biológicos de Orius insidiosus (Say, 1832) (Hemiptera, Anthocoridae). Revista Brasileira de Entomologia, v.47, n.1, p.19-24, 2003.

MENDES, S.M.; BUENO, V.H.P. Biologia de Orius insidiosus (Say) (Hemiptera: Anthocoridae) alimentado com Caliothrips phaseoli (Hood) (Thysanoptera: Thripidae). Neotropical Entomology, v.30, n.3, p.423428, 2001.

OLIVEIRA J.E.M.; DE BORTOLI, S.A.; SANTOS, R.F. Metodologia de criação do pulgão-do-algodoeiro Aphis gossypii Glover, 1877 (Hemiptera: Aphididae) em laboratório. Arquivos do Instituto Biológico, São Paulo, v.72, p.49, 2005. Suplemento 2. Trabalho apresentado na REUNIÃO ANUAL DO INSTITUTO BIOLÓGICO, 18., 2005, São Paulo, SP. Resumos. São Paulo: 2005. Resumo 062.

PEÑA-MARTINEZ, R. Identificacion de afidos de importancia agricola. In: URIAS-M, C.; RODRÍGUEZM, R.; ALEJANDRE, A, T. (Ed.). Afidos como vectores de virus en México. Montecillo: Centro de Fitopatologia, Montecillo, 1992. v.2, 135p. 
REZENDE, M.F.O. Biologia e consumo alimentar de Orius insidiosus (Say, 1832) (Hemiptera: Anthocoridae) sobre duas presas diferentes. 1990. 73f. Dissertação (Mestrado em Fitossanidade) - Universidade Federal de Lavras, Lavras, 1990.

RICHARDS, P.C.; SCHMIDT, J. The effect of selected dietary supplements on survival and reproduction of Orius insidiosus (Say) (Hemiptera: Anthocoridae). Canadian Entomologist, v.128, n.1, p.171-176, 1996.

SCHMIDT, J.M.; RICHARDS, P.C.; NADEL, H.; FERGUNSON, G.A. A rearing method for the production of large numbers of the insidiosus flower bug, Orius insidiosus (Say) (Hemiptera: Anthocoridae). Canadian Entomologist, v.127, n.3, p.445-447, 1995.

SILVEIRA, L.C.P.; BUENO, V.H. Orius insidiosus (Say, 1832) (Heteroptera: Anthocoridae): sensibilidade ao fotoperíodo e diapausa reprodutiva? Revista Brasileira de Entomologia, v.47, n.4, p.631-635, 2003.

SILVEIRA, L.C.P.; BUENO, V.H.; PIERRE, L.S.R.; MENDES, S.M. Plantas cultivadas e invasoras como habitat para predadores do gênero Orius (Wolff) (Heteroptera: Anthocoridae). Bragantia, v.62, n.2, p.261265, 2003.

SOUTHWOOD, T.R.E. Ecological methods with particular reference to the study of insect populations. 2.ed. London: Chapman and Hall, 1978. 524p.

STUDEBAKER, G.E.; KRING, T.J. Effects of insecticides on Orius insidiosus (Hemiptera: Anthocoridae), measured by field, greenhouse and petri dish bioassays. Florida Entomologist, v.82, n.2, p.178-185, 2003.

TOMMASINI, M.G.; VAN LENTEREN, J.C.; BURGIO, G. Biological traits and predation capacity of four Orius species on two prey species. Bulletin of Insectology, v.57, n.2, p.79-93, 2004.

Recebido em 24/5/06

Aceito em 28/3/08 